Preprint typeset in JHEP style - HYPER VERSION

\title{
On Non-Linear Actions for Massive Gravity
}

\author{
S. F. Hassan \\ Department of Physics $\&$ The Oskar Klein Centre, \\ Stockholm University, AlbaNova University Centre, SE-106 91 Stockholm, Sweden \\ E-mail: fawad@fysik.su.se \\ Rachel A. Rosen \\ Department of Physics 8 The Oskar Klein Centre, \\ Stockholm University, AlbaNova University Centre, SE-106 91 Stockholm, Sweden \\ E-mail: rarosen@fysik.su.se
}

\begin{abstract}
In this work we present a systematic construction of the potentially ghostfree non-linear massive gravity actions. The most general action can be regarded as a 2-parameter deformation of a minimal massive action. Further extensions vanish in 4 dimensions. The general mass term is constructed in terms of a "deformed" determinant from which this property can clearly be seen. In addition, our formulation identifies nondynamical terms that appear in previous constructions and which do not contribute to the equations of motion. We elaborate on the formal structure of these theories as well as some of their implications.
\end{abstract}

KEYWORDS: massive gravity. 


\section{Contents}

1. Introduction and Summary of Results 1

2. General Structure of non-Linear Massive Gravity 3

2.1 The Fierz-Pauli mass in linear theory

2.2 The auxiliary metric and the inverse metric

2.3 Non-linear generalizations of the Fierz-Pauli mass

3. The Minimal non-Linear Massive Gravity Action

3.1 The mass term as a minimally deformed determinant

3.2 Equations of motion 10

3.3 The screening solutions 11

4. The General non-Linear Massive Action 13

4.1 The generalized mass term as a deformed determinant 13

4.2 Formal construction 14

4.3 Equivalent descriptions of the action 16

5. Discussion of results and conclusions 18

\section{Introduction and Summary of Results}

There are several motivations for studying massive gravity. From the observational point of view, the conclusion that $95 \%$ of the universe is made of dark energy and dark matter is closely related to the assumption that general relativity is equally valid at all length scales. It is possible that modifications of general relativity over large distances change this picture and massive gravity is one such modification. It is also possible that a graviton mass ameliorates the cosmological constant problem. From the theoretical perspective, the shear difficulty of constructing a consistent theory of massive gravity makes the problem more interesting.

In 1939, Fierz and Pauli [1, 2] constructed the ghost-free theory of non-interacting massive gravitons in a flat background. Since then, this theory has stubbornly resisted a non-linear generalization. The work of Boulware and Deser [3] showed that a generic extension of the Fierz-Pauli (FP) theory to curved backgrounds will contain ghost instabilities. The ghost analysis in massive gravity was greatly simplified by the work of [4, motivated by the Goldstone-vector boson equivalence theorem in quantum field theory. The authors in [4] argued that, since a graviton mass can be associated with a spontaneous breaking of general covariance, the associated Goldstone modes in a non-unitary gauge will contain 
the ghost information. Based on this observation, a procedure was outlined in 4 , 5] where the ghost could be avoided order-by-order by tuning the coefficients in an expansion of the mass term in powers of the metric perturbation $h_{\mu \nu}$ and the longitudinal Goldstone mode $\pi$. An added advantage is that within this effective field theory-like framework, if a ghost turns out to be heavier than the UV cutoff of the effective theory it can be safely ignored. Thus even when the ghost is not completely avoidable, it could still be manageable. The preliminary conclusion in [5] was that at the quartic order in Goldstone modes, the expansion will unavoidably contain ghosts.

This problem was recently revisited by de Rham and Gabadadze [6] who showed that massive gravity ghosts can indeed be avoided, or at least their appearance relegated to higher energy scales where the effective field theory may no longer be applicable. One can then successfully construct a theory that is ghost-free in the so-called decoupling limit, which amounts to retaining terms only first order in $h_{\mu \nu}$, but all orders in $\pi$ [6, 7]. In particular, [7] constructed a non-linear massive action starting from the decoupling limit data. Using this theory it becomes possible to study the ghost away from the decoupling limit. It was concluded that one of the resummed theories is ghost-free at least up to quartic order in the nonlinearities.

In this work we present a systematic construction of massive gravity actions that simplifies the analysis and brings out several important features. The main results are summarized below. The implications of these results are discussed in more detail in the concluding section.

- We construct the most general, potentially ghost-free, mass term as a "deformed" determinant. The general massive gravity action can be regarded as a 2-parameter deformation of a minimal massive action which is, in turn, the minimal non-linear extension of Fierz-Pauli massive gravity. The origin of the 2 parameters can be easily seen in this formulation. No further generalizations exist in 4 dimensions. The full nonlinear equations of motion are also written down.

- It is shown that the earlier constructions of non-linear massive actions lead to nondynamical terms in the action that do not contribute to the equations of motion. These terms arise due to an assumption made to simplify the construction. In our formulation it becomes straightforward to isolate and remove these terms, implying that the resummed theory found in [7] has an equivalent description in terms of a simpler action. This also shows that while the massive action was obtained starting with a covariant version of the Fierz-Pauli mass, it also turns out to describe theories which reduce to the non-covariant Fierz-Pauli mass at lowest order and, hence, is more general than it seems.

- We emphasize the physical relevance of generalizing the auxiliary metric $\eta_{\mu \nu}$, used in the construction, to a general $f_{\mu \nu}$. In this context, we discuss solutions of the minimal theory that have the potential to avoid the observational consequences of the vDVZ discontinuity [8, 9] as well as screen a large cosmological constant. 
The actions presented here are known to be ghost-free at the interactive level to first order in the metric perturbation $h_{\mu \nu}$ and to all orders in the Goldstone longitudinal mode, $\pi$. This was extended to quartic order by an ADM analysis in [7]. However, a proof that these actions are ghost free at the complete non-linear level is still lacking (see, for example, [10] for an argument that the ghost is in fact present at the quartic order). But the stronger statement is that if any such non-linear ghost free action exists, it will have to belong to this family of actions (see note added at the end).

The organization of this paper is as follows. In section 2 we review the basic features of massive gravity, paying particular attention to the features that will be most relevant when constructing a nonlinear theory. We also briefly summarize the recent developments in constructing ghost-free nonlinear extensions of the FP action. In section 3 we construct the minimal non-linear extension of the Fierz-Pauli mass term and discuss its screening solutions as well as the origin of non-dynamical terms. In section 4 the generalization of this model is considered. We describe the formal structure of the theory and argue that there exists at most a 2-parameter family of actions with the desired properties. We write down the full non-linear equations of motion for the generalized theory. Some implications of our results are discussed in section 5 .

\section{General Structure of non-Linear Massive Gravity}

In this section we review features of massive gravity that are relevant to the construction of non-linear mass terms, and make some observations that clarify the underlying structures. We then briefly discuss recent developments in constructing ghost-free non-linear extensions of Fierz-Pauli massive gravity. This provides the background and conventions for the systematic approach of the following sections.

\subsection{The Fierz-Pauli mass in linear theory}

Fierz and Pauli [1, 2] constructed a mass term for linearized metric fluctuations around flat spacetime, $h_{\mu \nu}=g_{\mu \nu}-\eta_{\mu \nu}$,

$$
\frac{m^{2}}{4}\left(h_{\mu \nu} h^{\mu \nu}-a h_{\mu}^{\mu} h_{\nu}^{\nu}\right), \quad \text { with } \quad a=1
$$

For any other value of $a$ the theory has a ghost. Invariance under infinitesimal general coordinate transformations (GCT) can be restored by introducing Stückelberg fields and replacing $h_{\mu \nu}$ by $h_{\mu \nu}+\partial_{\mu} \pi_{\nu}+\partial_{\nu} \pi_{\mu}$. In the Stückelberg formulation, the longitudinal mode $\pi$ defined through $\pi_{\mu}=\pi_{\mu}^{\perp}+\partial_{\mu} \pi$, appears in the action through a 4-derivative term and carries the ghost degree of freedom. The ghost decouples only for $a=1$. Unfortunately, unlike the case of massless linearized general relativity, there is no obvious unique way to extend the massive linearized theory to the non-linear level. But in recent work, ghost decoupling has turned out to be a powerful criterion. Let us first discuss the general features of a non-linear mass term before discussing the recent developments towards its construction. 


\subsection{The auxiliary metric and the inverse metric}

On general grounds, a non-linear mass term in gravity (a) cannot contain derivatives of $g_{\mu \nu}$ and (b) should be invariant under general coordinate transformations (GCT). Such a term cannot be constructed using the metric alone since the only two relevant quantities, $\operatorname{det} g$ and $\operatorname{Tr} g=4$, are not adequate for this purpose [3]. To overcome this, one of the following options may be used:

1. Introduce an extra rank two tensor $f_{\mu \nu}$ as in [3, 44.

2. Introduce an auxiliary extra dimension [11, 12].

3. Generate a mass through a gravitational Higgs mechanism ${ }^{1}$ [13, 14, 15, 16, 17, 18].

Both options (2) and (3) can be formulated in terms of (1) as will be discussed below for Higgs gravity and in [19] for the extra auxiliary dimension model. Hence we will concentrate on this first case.

Combining the dynamical metric $g_{\mu \nu}$ with a covariant rank two tensor $f_{\mu \nu}(x)$ (henceforth called the auxiliary metric), one can construct arbitrary scalar functions $F\left(g^{-1} f\right)$ and $F^{\prime}\left(f^{-1} g\right)$. The GCT invariant mass term has to be written in terms such functions. A special case is when the mass term involves only $F\left(g^{-1} f\right)$, i.e., it depends only on the inverse metric $g^{\mu \nu}$. A simple inspection shows that the massive gravity actions considered in [4], and in the subsequent works [5, 6, 7], all belong to this class ${ }^{2}$, even though, in practice, the setups may have been described in terms of $g_{\mu \nu}$. In the last paragraph of this section we argue that the combination $g^{-1} f$ is adequate to describe any ghost free mass term and so will be explicitly assumed here. Thus we consider massive gravity actions of the form,

$$
S=-M_{p}^{2} \int d^{4} x \sqrt{-g} R(g)+M_{p}^{2} m^{2} \int d^{4} x \sqrt{-g} F\left(g^{-1} f\right) .
$$

Invariance under general coordinate transformations implies that $F$ must be expandable as a sum of products of the type $\operatorname{Tr}\left[\left(g^{-1} f\right)^{n}\right]$.

Invariance under GCT is insured since $f_{\mu \nu}$ transforms as a rank 2 tensor. To make this explicit, one can parametrize the auxiliary metric as a coordinate transformation of some fixed metric [4],

$$
f_{\mu \nu}(x)=\frac{\partial \phi^{a}}{\partial x^{\mu}} \bar{f}_{a b}(\phi) \frac{\partial \phi^{b}}{\partial x^{\nu}}
$$

The $\phi^{a}(x)$ are to be regarded as general coordinate transformations of the $x^{\mu}$ and generalize Stückelberg fields to non-linear level. $\bar{f}_{a b}$ is a fixed auxiliary metric that should be chosen

\footnotetext{
${ }^{1}$ Note that in this context, the term Higgs mechanism does not imply the existence of a mass generation mechanism on par with that in vector gauge theories, and the corresponding ramifications for unitarity, etc. Here, it mainly refers to the association of the graviton mass with scalars of the form $\phi^{\mu}=x^{\mu}+\delta \phi$. We retain the terminology with this caveat.

${ }^{2}$ This also holds for the "auxiliary extra dimension" model of [11, 12, 20] which is special in that it admits a description in terms of both $g^{-1} f$ and $f^{-1} g$ [19.
} 
consistent with the physics of the problem. Both these are scalar functions ${ }^{3}$ of $x^{\mu}(\bar{f}$ is a tensor with respect to reparametrizations of $\phi^{a}$ ). The physical (unitary) gauge corresponds to choosing $\phi^{a}(x)=x^{a}$. In [3] $\bar{f}$ is identified with a background dynamical metric $\bar{g}$ in the physical gauge. If $\bar{f}$ is taken to be a flat metric, the $\phi^{a}$ can be chosen such that $\bar{f}_{a b}=\eta_{a b}$.

\subsection{Non-linear generalizations of the Fierz-Pauli mass}

Let us now describe the ideas that have recently been used to construct non-linear graviton mass terms ${ }^{4}$. Given the auxiliary metric $f_{\mu \nu}$, a covariant non-linear version of the FierzPauli mass (2.1) can be constructed in terms of a matrix $H_{\nu}^{\mu}$ given by,

$$
g^{\mu \lambda} f_{\lambda \nu}=\delta_{\nu}^{\mu}-H_{\nu}^{\mu}
$$

This is obviously a $(1,1)$ tensor under GCT. Note that, rewritten as $H_{\mu \nu} \equiv g_{\mu \lambda} H_{\nu}^{\lambda}=$ $g_{\mu \nu}-f_{\mu \nu}$, one recovers the matrix introduced in 泪 in a more elaborate setup. Any mass term can be expressed entirely in terms of $H_{\nu}^{\mu}$, and we will only retain this in the description. Then the Fierz-Pauli mass can be generalized to a covariant non-linear form (see [4] and references therein),

$$
\frac{m^{2}}{4} \sqrt{-g}\left(H_{\nu}^{\mu} H_{\mu}^{\nu}-H_{\mu}^{\mu} H_{\nu}^{\nu}\right)
$$

which reduces to the original FP mass (2.1) at the quadratic order in $h_{\mu \nu}$ and in the unitary gauge for $\phi^{a}$. We refer to this as the covariant FP term.

But this is not enough. Though (2.5) is ghost free at the quadratic order around flat background, the ghost mode generally reappears at the quadratic order around curved backgrounds [3]. Equivalently stated, the ghost reappears at higher orders around flat background. To cure this problem, the idea, as proposed in [4] and pursued in [5, 6], is to add terms to (2.5) that are higher order in $H_{\nu}^{\mu}$ to cancel the ghost at higher orders.

To simplify the ghost analysis, the theory is often written as an expansion around flat background, which for $g^{\mu \nu}$ gives,

$$
g^{\mu \nu}=\eta^{\mu \nu}+\mathfrak{h}^{\mu \nu} .
$$

In terms of $h_{\mu \nu}=g_{\mu \nu}-\eta_{\mu \nu}$, one has $\mathfrak{h}^{\mu \nu}=\sum_{n=1}^{\infty}\left[\left(-\eta^{-1} h\right)^{n} \eta^{-1}\right]^{\mu \nu}$. Also, since the $\phi^{a}(x)$ are general coordinate transformations of $x^{\mu}$, they can be expanded around the "unit transformation" as 泪,

$$
\phi^{\mu}(x)=x^{\mu}+\pi^{\mu}(x) .
$$

The $\pi^{\mu}$ transform as "coordinate differences" under GCT. Then $H_{\nu}^{\mu}$ given by (2.4) take the form (on choosing $\bar{f}_{\mu \nu}=\eta_{\mu \nu}$ to facilitate comparison with FP theory),

$$
H_{\nu}^{\mu}=-\partial^{\mu} \pi_{\nu}-\partial_{\nu} \pi^{\mu}-\partial^{\mu} \pi_{a} \partial_{\nu} \pi^{a}-\mathfrak{h}_{\rho}^{\mu}\left(\delta_{\nu}^{\rho}+\partial_{\nu} \pi^{\rho}+\partial^{\rho} \pi_{\nu}+\partial^{\rho} \pi_{a} \partial_{\nu} \pi^{a}\right)
$$

\footnotetext{
${ }^{3}$ To see this, start with a coordinate system $\left\{\phi^{\mu}\right\}$ and consider two reparametrizations $\left\{x^{\mu}\right\}$ and $\left\{x^{\prime \mu}\right\}$ defined by $\phi^{\mu}(x)$ and $\phi^{\prime \mu}\left(x^{\prime}\right)$. Now, a coordinate transformation from $x$ to $x^{\prime}$ is subject to $\phi^{\mu}(x)=\phi^{\prime \mu}\left(x^{\prime}\right)$ clarifying that $\phi^{\mu}(x)$ are scalars.

${ }^{4}$ While the structures introduced in this subsection are useful for motivating the form of the nonlinear actions of later sections, and for seeing the relation with other recent work, they are not needed for formulating the non-linear theory, which is good news in view of footnote
} 
Here, all indices have been raised or lowered with respect to their natural positions with the background metric $\eta_{\mu \nu}$. The expression is exact in $\pi^{\mu}$ and in $\mathfrak{h}^{\mu \nu}$ which, so far, need not be infinitesimal. If the graviton mass is assumed to arise as a result of a spontaneous breaking of GCT, through some kind of gravitational Higgs mechanism, then the $\pi^{a}$ would be the unphysical (pure gauge) Goldstone modes associated with the symmetry breaking 13, 14.

The field most relevant to the ghost problem is the "longitudinal" mode $\pi$, given by,

$$
\pi_{\mu}=\pi_{\mu}^{\perp}+\partial_{\mu} \pi
$$

Let's use the notation $H$ for the matrix with elements $H_{\nu}^{\mu}$ to avoid mixup with $H=H_{\mu}^{\mu}$. Also, let $\Pi$ denote the matrix with elements $\Pi_{\nu}^{\mu}=\partial^{\mu} \partial_{\nu} \pi$. Then retaining only $\pi$, and setting $\pi_{\mu}^{\perp}=0$, one has,

$$
g^{-1} f \equiv(\mathbb{1}-\mathbb{H})=(\mathbb{1}+\mathfrak{h} \eta)(\mathbb{1}+\Pi)^{2} .
$$

This expression is exact ${ }^{5}$ to all orders in $\mathfrak{h}$ and $\pi$.

Recent developments: As discussed in 4 , 旬, the Boulware-Deser ghost instability of massive gravity is associated with the appearance of $\pi$ dependent terms in the action with more than 2 time derivatives. Thus, in the massive gravity action (2.2), an expansion of $F(\mathbb{1}-\mathbb{H})$ in powers of $\mathbb{H}$ should be constructed in such a way as to avoid this problem. Such a program was carried out successfully by de Rham and Gabadadze in [6]. They obtained a 2 parameter family of actions to quintic order in $\mathbb{H}$ that avoids the ghost problem at least up to first order in $h_{\mu \nu}$ and all orders in $\pi$. Subsequently [7] proposed a method for resumming such expansions in powers of $\mathbb{H}$ into closed-form non-linear actions.

In the next two sections, we present an alternative formulation of the massive action based on a "deformed determinant" that clearly brings out the systematics, showing in particular that the framework is more general than might appear at first sight.

Adequacy of the description in terms of $g^{-1} f$ : Consider a mass term that also depends on $f^{-1} g \equiv(\mathbb{1}-\mathbb{H})^{-1}$. It is obvious that any scalar function $F^{\prime}\left(f^{-1} g\right)$ can also be expanded in powers of $H$. The quintic order polynomials of $H$ analyzed in [6] are general enough that they do not exclude $F^{\prime}$ in the mass term. However, as can be seem from this work as well as from [7], all ghost free combinations are obtainable from actions that contain $F\left(g^{-1} f\right)$ alone, owing to the simple relation between $\Pi$ and $H$ in $(2.9)$. Hence this structure is adequate for describing ghost free massive gravity actions. Though, up to a given order, the $\mathrm{H}$-expansions may also be obtainable from an $F^{\prime}\left(f^{-1} g\right)$, that would correspond to a complicated rewriting of $F\left(g^{-1} f\right)$ to that order.

\footnotetext{
${ }^{5} \mathrm{~A}$ word caution on covariance issues: The above parametrization of fluctuations refers to a perturbative expansion around flat background. At the non-linear level the $\pi^{\mu}$ are coordinate differences and hence $\pi_{\mu}^{\perp}$ and $\pi$ are not proper vectors and scalars. Thus, strictly speaking, statements that hold for vector and scalar modes at the linearized level, may not fully apply here. Also, the resulting expressions cannot be covariantized by simply using covariant derivatives. If one insists on using this type of parametrization for the non-linear theory, while maintaining manifest general covariance, then an option is to use Riemann normal coordinates and express $\pi^{\mu}$ in terms of a geodesic tangent vector $u^{\mu}$ and derivatives of the curvature tensor as in 21 .
} 
Relation to Higgs gravity: In Higgs gravity setups [15, 16, 17, 18], one considers gravity coupled to 4 scalar fields, say $\phi^{a}$ with a Minkowskian internal metric. The scalars are supposed to acquire a vacuum expectation value $\left\langle\phi^{a}\right\rangle=x^{a}$ through some dynamics and break the GCT symmetry of the action, rendering the gravitons massive ${ }^{6}$. Note that all terms involving derivatives of the $\phi^{a}$ can be written in terms of $g^{-1} f$, or using the trace structure, in terms of $\partial_{\mu} \phi^{a} g^{\mu \nu} \partial_{\nu} \phi^{b} \bar{f}_{b c}$. For example, the basic kinetic term is $\operatorname{Tr}\left(g^{-1} f\right)$. This accounts for the practical similarity between Higgs gravity and massive gravity, as, for example, in [23].

\section{The Minimal non-Linear Massive Gravity Action}

In this section we construct a minimal non-linear extension of the Fierz-Pauli mass term and discuss some of its main features. This is useful for understanding the more general non-linear extension to be given in the next section.

\subsection{The mass term as a minimally deformed determinant}

The construction of the non-linear graviton mass initiated in [4, 5] and successfully employed in [6, 7], is based on two simple criteria.

1. The absence of ghosts at low orders: The function $F\left(g^{-1} f\right)$ in the massive gravity action (2.2) is constrained by the requirement that its expansion around a flat background leads to a ghost-free theory. The expansion involves $\mathfrak{h}^{\mu \nu}$ and $\pi_{\mu}=\partial_{\mu} \pi$ (ignoring the "vector" modes) through (2.9). As explained in [5], the ghost is associated with $\pi$ terms with higher than two derivatives which, therefore, should not arise in the expansion of $F$. In particular, the $\mathfrak{h}$-independent part, $\left.F\left(g^{-1} f\right)\right|_{h=0}$, should contain the potentially dangerous $\pi$ terms only through total derivatives. The next step is to check that the term linear in $\mathfrak{h}$ is also ghost free (this is, of course, necessary but not sufficient for avoiding the ghost to higher orders in $\mathfrak{h})$.

2. The recovery of the covariant Fierz-Pauli action (2.5) at the lowest order.

Note that the second criterion demands more than the minimum requirement of reproducing the original FP mass in flat background (2.1). Hence it could, potentially, exclude non-linear actions that reduce to (2.1) without producing (2.5). We will show that these two classes of actions only differ by non-dynamical terms that can be easily isolated.

In [5, 6, 7], to implement the first criterion, total derivative functions of $\Pi_{\nu}^{\mu}=\partial^{\mu} \partial_{\nu} \pi$ are explicitly constructed in terms of traces of powers of $\Pi(2.9)$. In contrast, the approach here is based on the observation that all such total derivative terms naturally appear in the expansion of $\operatorname{det}(\mathbb{1}+\Pi)$. This simplifies both the procedure and the final results by identifying the redundancies that result from the second criterion.

\footnotetext{
${ }^{6}$ A dynamical realization of Higgs gravity is provided by the "brane induced gravity" construction, as discussed in 22]. While the obvious breaking of translation invariance in directions transverse to the brane generates massless Goldstone modes, a more subtle spontaneous breaking of GCT along the brane occurs as a result of integrating out bulk modes. This automatically generates the $\phi^{a}$ and the graviton mass in a gauge invariant set up, though a ghost free construction is still lacking.
} 
To satisfy the first criterion, consider $F\left(g^{-1} f\right)$ at lowest order, i.e., for $\mathfrak{h}=0$, when,

$$
\left.\left(g^{-1} f\right)\right|_{h=0}=(\mathbb{1}+\Pi)^{2} .
$$

As mentioned above, at this order $\pi$ must appear in the Lagrangian only in total derivative terms. All such total derivative terms appear automatically (with given coefficients) in an expansion of the determinant,

$$
\operatorname{det}(\mathbb{1}+\Pi)=\sum_{n=0}^{4} \frac{-1}{n !(4-n) !} \epsilon_{\mu_{1} \cdots \mu_{n} \lambda_{n+1} \cdots \lambda_{4}} \epsilon^{\nu_{1} \cdots \nu_{n} \lambda_{n+1} \cdots \lambda_{4}} \partial^{\mu_{1}} \partial_{\nu_{1}} \pi \cdots \partial^{\mu_{n}} \partial_{\nu_{n}} \pi .
$$

The total derivative nature is manifest due to the antisymmetry property of the epsilon tensor. We can choose $\left.F\right|_{h=0}$ to have this form, but the first two terms of the summation, $1+\partial^{\mu} \partial_{\mu} \pi$, (for $n=0$ and 1, respectively) cannot arise in an action that, by the second criterion, reduces to the covariant FP form (2.5) at the lowest order (since this is at least quadratic in $\pi$ ). Thus choosing $\left.F\right|_{h=0}$ consistent with the second criterion amounts to $\operatorname{deforming} \operatorname{det}(\mathbb{1}+\Pi)$ by removing the first two terms from the above expansion,

$$
\left.F\left(g^{-1} f\right)\right|_{h=0} \sim \operatorname{det}(\mathbb{1}+\Pi)-\operatorname{Tr}(\mathbb{1}+\Pi)+3 .
$$

This is the minimal deformation of the determinant structure. One may also consider non-minimal deformations by changing the coefficients of the $n>2$ terms in (3.2) which, obviously, still preserve their total derivative structure. Here we concentrate on the minimal deformation, leaving the general case to the next section.

Now, the field $\pi$ enters the action only through $g^{-1} f$, in the combination (2.9). In particular, $\operatorname{det}(\mathbb{1}+\Pi)=\left.\sqrt{\operatorname{det}\left(g^{-1} f\right)}\right|_{h=0}$ and $\operatorname{Tr}(\mathbb{1}+\Pi)=\left.\operatorname{Tr} \sqrt{g^{-1} f}\right|_{h=0}$. Thus, going beyond $\mathfrak{h}=0$ requires making the substitution $\mathbb{1}+\Pi \rightarrow \sqrt{g^{-1} f}$ leading to a massive gravity action (2.2), with the non-linear mass term

$$
m^{2} \int d^{4} x \sqrt{-g} F=2 m^{2} \int d^{4} x \sqrt{-g}\left[\sqrt{\operatorname{det}\left(g^{-1} f\right)}-\operatorname{Tr} \sqrt{g^{-1} f}+3\right] .
$$

The construction involves the square root matrix defined such that ${ }^{7} \sqrt{E} \sqrt{E}=E$. Again, the mass term is given by a minimal deformation of $\operatorname{det}\left(\sqrt{g^{-1} f}\right)=\operatorname{det}\left(\mathbb{1}+\sqrt{g^{-1} f}-\mathbb{1}\right)$ that involves removing the first two terms from its expansion.

To confirm that our massive action is the non-linear completion of one of the ghost free quintic order polynomial actions of [6], we expand it in powers of $\mathrm{H}$. To lowest order

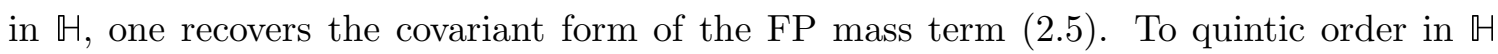
one obtains the action presented in [6] with the particular coefficients ${ }^{8} c_{3}=\frac{1}{6}, d_{5}=-\frac{1}{48}$ ( $f_{7}$ there multiplies terms that vanish in 4-dimensions as will be discussed in section 4 ). Generalizations of this action are considered in the next section.

The above action can be trimmed further:

\footnotetext{
${ }^{7}$ For the metric, we stick to the conventional notation $\sqrt{-g}=\sqrt{-\operatorname{det} g}$. In all other cases, $\sqrt{E}$ represents the square-root matrix and not the determinant.

${ }^{8}$ To correctly match the coefficients at quintic order we note that there is a typo in [6]. The expression for $f_{3}$ should be corrected to $f_{3}=\frac{3}{8} c_{3}-3 d_{5}+20 f_{7}$.
} 
1. The term $3 \sqrt{-g}$ is needed to cancel out a cosmological constant of order $M_{p}^{2} m^{2}$. If this cancellation is not required, the factor 3 can be replaced by a free parameter.

2. The first term simply reduces to $\sqrt{-\operatorname{det} f}$ and does not contribute to the metric equation of motion. As shown in the next subsection, it also does not contribute to the $\phi^{a}$ equations since these are already contained in the metric equation. Thus, being non-dynamical, it can be dropped from the action at the classical level (as long as one does not intend to promote $\bar{f}_{\mu \nu}$ to a dynamical variable). The origin of this term will be explained below.

This leaves us with an action that turns out to be the minimal non-linear extension of the massive Fierz-Pauli action in the presence of a cosmological constant $\Lambda=\Lambda^{\prime}+6 m^{2}$,

$$
S_{\min }=-M_{p}^{2} \int d^{4} x \sqrt{-g}\left[R+2 m^{2} \operatorname{Tr} \sqrt{g^{-1} f}+\Lambda^{\prime}\right] .
$$

Note that although the action is constructed as a minimal deformation of a determinant, the determinant itself becomes non-dynamical and only the "deformation" stays on. This action is also "minimal" in the sense of having the simplest structure as compared to the generalizations constructed in the next section.

If we now expand the mass term (3.5) in powers of $\mathbb{H}$ we find, to quadratic order,

$$
2 m^{2} \sqrt{g}\left(1-\frac{1}{2} \operatorname{Tr} \boxplus-\frac{1}{8} \operatorname{Tr}\left(\uplus^{2}\right)+\mathcal{O}\left(\uplus^{3}\right)\right),
$$

which is not of the covariant FP form (2.5). However, expanding this in the metric perturbation $h_{\mu \nu}$ around flat spacetime does reproduce the basic FP form (2.1). Hence, this is a valid mass term. What has happened is as follows: As stated above, the first term $\sqrt{-\operatorname{det} f}$ in (3.4) does not contribute to the equations of motion. However, this term is needed to reproduce the expansion that starts with the covariant FP form (2.5) at the quadratic level, as was required by the construction. But this requirement can be relaxed since it is totally acceptable that a non-linear massive action directly reduces to the basic Fierz-Pauli form (2.1) at the quadratic level in $h_{\mu \nu}$ without ever reproducing (2.5). Thus, one concludes that while taking the covariant FP term (2.5) as the starting point simplifies the construction, it does not make it less general. Rather, it only leads to redundant non-dynamical terms in the action that are present for "cosmetic" reasons. The formulation presented here makes this manifest and conveniently isolates the redundant terms. To drive home the point, note that in a formulation that does not take care of the redundancy, the same minimal theory looks much more complicated than (3.5), i.e., it is given by (3.4) where $\sqrt{\operatorname{det}\left(g^{-1} f\right)}$ is expanded in traces of powers of the square-root matrix $\sqrt{g^{-1} f}$.

Returning to the ghost issue, when expanded in powers of $\mathfrak{h}$ around flat background, the lowest order term in (3.4) or (3.5) is ghost free by construction. The next term, linear in $\mathfrak{h}$ and to all orders in $\pi$, is also healthy having the form (see footnote 9 below),

$$
\frac{1}{2} m^{2} \mathfrak{h}^{\mu \nu}\left(\partial_{\mu} \partial_{\nu}-\eta_{\mu \nu} \square\right) \pi
$$


In particular no higher powers and derivatives of $\pi$ arise at the linear order in $\mathfrak{h}$. This corresponds to one of the models constructed in [6] to quintic order in $\mathbb{H}=\mathbb{1}-g^{-1} f$. It was pointed out there that, in the absence of non-linear $\pi$ interactions, the theory will not exhibit the Vainshtein mechanism [24, 25] which is associated with the strong coupling behavior of $\pi$ in the limit $m \rightarrow 0$. More precisely, non-linear $\pi$ terms at higher orders in $\mathfrak{h}$ may still generate a strong coupling scale but this will be suppressed by the Planck scale, leading to a much smaller Vainshtein radius. The issue of ghosts at higher orders in $\mathfrak{h}$ is still an open question and will not be addressed here.

\subsection{Equations of motion}

In this subsection we write down the $g^{\mu \nu}$ and $\phi^{a}$ equations of motion and, in particular, show that the "cosmetic" $\sqrt{-\operatorname{det} f}$ term in (3.4) does not contribute to the $\phi^{a}$ equations.

The metric equation: This can be worked out using (3.5) and reads ${ }^{9}$,

$$
\begin{aligned}
R_{\mu \nu}-\frac{1}{2} g_{\mu \nu} R-\frac{1}{2} \Lambda g_{\mu \nu}+\frac{1}{2} & m^{2}\left[g \sqrt{g^{-1} f}+\left(\sqrt{g^{-1} f}\right)^{T} g\right]_{\mu \nu} \\
& +m^{2} g_{\mu \nu}\left(3-\operatorname{Tr} \sqrt{g^{-1} f}\right)=G_{N} T_{\mu \nu} .
\end{aligned}
$$

At least for a diagonal metric ansatz, as in [26], the square-root matrix simplifies and one gets closed-form non-linear differential equations that could in principle be solved.

The left hand side is not automatically divergence free, rather its divergence yields,

$$
\nabla_{\mu}\left(\sqrt{g^{-1} f}\right)_{\nu}^{\mu}+g^{\mu \rho} g_{\nu \sigma} \nabla_{\mu}\left(\sqrt{g^{-1} f}\right)_{\rho}^{\sigma}-2 \partial_{\nu} \operatorname{Tr}\left(\sqrt{g^{-1} f}\right)=0 .
$$

Here the covariant derivatives are to be written out regarding $\sqrt{g^{-1} f}$ as a $(1,1)$ tensor, similar to $g^{-1} f$. This follows from the expansion of the radical in powers of $\mathbb{H}$, or from the fact that, using $E=(\sqrt{E})^{2}$, a transformation $E \rightarrow A E A^{-1}$ implies $\sqrt{E} \rightarrow A \sqrt{E} A^{-1}$. The trace of (3.8) gives,

$$
R+2 \Lambda+3 m^{2}\left(\operatorname{Tr} \sqrt{g^{-1} f}-4\right)=G_{N} T_{\mu}^{\mu} .
$$

A simple class of solutions will be considered below. It is instructive to contrast these equations with their linearized forms around flat spacetime (the FP case). The linear version of (3.9) implies the vanishing of the linearized curvature scalar $R_{L}$. A non-zero $R_{L}$ cannot be recovered in the limit $m^{2} \rightarrow 0$ which is at the origin of the vDVZ discontinuity of linearized massive gravity.

The $\phi^{a}$ equations: These are equivalent to the divergence of the metric equation (3.9). This is a consequence of the general covariance of the action $S_{\min }[g, \phi]$. Indeed, under a general coordinate transformation, $\delta x^{\mu}=\xi^{\mu}, \delta g^{\mu \nu}=-2 \nabla^{(\mu} \xi^{\nu)}$ and $\delta \phi^{a}=-\xi^{\mu} \partial_{\mu} \phi^{a}$. The invariance of the action then implies $\int d^{4} x \sqrt{-g}\left[\left(\frac{2}{\sqrt{-g}} \frac{\delta S}{\delta g^{\mu \nu}}\right) \nabla^{\mu} \xi^{\nu}+\frac{1}{\sqrt{-g}} \frac{\delta S}{\delta \phi^{a}} \partial_{\nu} \phi^{a} \xi^{\nu}\right]=0$, or on integrating by parts,

$$
\nabla^{\mu}\left(\frac{2}{\sqrt{-g}} \frac{\delta S}{\delta g^{\mu \nu}}\right)=\frac{1}{\sqrt{-g}} \frac{\delta S}{\delta \phi^{a}} \partial_{\nu} \phi^{a} .
$$

${ }^{9}$ Note that $E=(\sqrt{E})^{2}$ implies $\delta E=(\delta \sqrt{E}) \sqrt{E}+\sqrt{E}(\delta \sqrt{E})$, so that $\operatorname{Tr}(\delta \sqrt{E})=(1 / 2) \operatorname{Tr}\left[(\sqrt{E})^{-1} \delta E\right]$. This last equation holds only under the trace. Then for $E=g^{-1} f$, a variation of the metric gives $\operatorname{Tr}\left(\delta \sqrt{\left.g^{-1} f\right)}=(1 / 4) \operatorname{Tr}\left[\left(g \sqrt{g^{-1} f}+\left(\sqrt{g^{-1} f}\right)^{T} g\right) \delta g^{-1}\right]\right.$, where use is made of the cyclic property of the trace and $f\left(\sqrt{g^{-1} f}\right)^{-1}=g\left(g^{-1} f\right)\left(\sqrt{g^{-1} f}\right)^{-1}=g \sqrt{g^{-1} f}$. The result is then explicitly symmetrized. 
The left hand side is the divergence of (3.8). Since $\phi^{a}(x)$ are non-singular coordinate transforms of $x^{\mu}$, the matrix $\partial_{\nu} \phi^{a}$ is invertible. Hence the $\phi^{a}$ equations $\delta S / \delta \phi^{a}=0$ are equivalent to the divergence equations (3.9). As a consequence, the $\phi^{a}$ equations are not affected by $\sqrt{-\operatorname{det} f}$ in (3.4), since it does not contribute to the metric equation. It is instructive to check this explicitly. The $\phi^{a}$ equations of (3.4), including $\sqrt{-\operatorname{det} f}$, are,

$$
\nabla_{\mu}\left(D^{\mu \nu} \partial_{\nu} \phi^{a} \bar{f}_{a c}\right)+D^{\mu \nu} \partial_{\nu} \phi^{a} \frac{\delta \bar{f}_{a b}}{\delta \phi^{c}} \partial_{\mu} \phi^{b}=0
$$

where,

$$
D^{\mu \nu}=\sqrt{\operatorname{det}\left(g^{-1} f\right)}\left(f^{-1}\right)^{\mu \nu}-\frac{1}{2}\left[\sqrt{g^{-1} f}+g^{-1}\left(\sqrt{g^{-1} f}\right)^{T} g\right]_{\rho}^{\mu}\left(f^{-1}\right)^{\rho \nu} .
$$

Multiplying by the invertible matrix $\partial_{\lambda} \phi^{c}$ and manipulating, this reduces to the equivalent expression (3.9), as expected. In particular, the contribution from the first term of $D^{\mu \nu}$ completely drops out, once again showing that $\sqrt{-\operatorname{det} f}$ is non-dynamical.

\subsection{The screening solutions}

For the massive gravity actions considered here, the proof of absence of ghosts at low orders is valid only for a flat auxiliary metric, $\bar{f}_{\mu \nu}=\eta_{\mu \nu}$. This theory exhibits the vDVZ discontinuity [8, 9] at the linear order which implies radically different bending of light near massive objects as compared to general relativity. This difference needs to be avoided at the non-linear level, at least in the vicinity of massive objects, should a theory of light gravitons describe the real world. In general, massive gravity can achieve this via the Vainshtein mechanism $^{10}$ [24, 25]. However, the analysis of [6], performed in the so-called decoupling limit approximation, indicates that this mechanism will not work precisely for the minimal model (3.5), as briefly discussed following equation (3.7). Besides this, the divergence conditions (3.9) are constraining enough that, when working in a physical gauge, they could rule out spacetimes of physical interest, including homogeneous, isotropic FRW cosmologies [28]. Some of these issues can already be seen in [29, 30]. Thus, the classical solutions in massive gravity models with flat $\bar{f}_{\mu \nu}$ may drastically differ from general relativity.

Of course, the action (3.5) can also be used for non-flat $\bar{f}$, although in this case, the ghost problem has to be investigated afresh. Let us split the metric into background and fluctuation parts, $g_{\mu \nu}=\hat{g}_{\mu \nu}+h_{\mu \nu}$ and choose the physical gauge, $\phi^{\mu}=x^{\mu}$. Now take,

$$
f_{\mu \nu}=c^{2} \hat{g}_{\mu \nu}
$$

The caveat here is that, classically, there is no real distinction between the "background" and the "fluctuation" (unless $T_{\mu \nu}$ has a natural split in this way). In principle, this problem can be avoided by giving appropriate dynamics to $f_{\mu \nu}$. We will not attempt that here,

\footnotetext{
${ }^{10}$ But even in that case, the vDVZ discontinuity can be tested far away from massive sources. For example, by comparing mass estimates for galaxies and galaxy clusters as derived from gravitational lensing, with dynamical mass estimates, such models can be constrained observationally [27].
} 
except to say that in that case the $\sqrt{-\operatorname{det} f}$ term can no longer be disregarded. If we simply add a term $M_{p}^{2} m^{2} \lambda_{f} \sqrt{-\operatorname{det} f}$ to the action, then the $f_{\mu \nu}$ minimization condition,

$$
\left(\sqrt{g^{-1} f}\right) f^{-1}+f^{-1}\left(\sqrt{g^{-1} f}\right)^{T}-2 \lambda_{f} \sqrt{\operatorname{det} g^{-1} f} f^{-1}=0
$$

has the solution $c=\left(1 / \lambda_{f}\right)^{\frac{1}{3}}$. More generally, the above ansatz naturally arises as a solution in bi-gravity theories [31, 32]. Here, we will not attempt giving $f_{\mu \nu}$ any specific dynamics, but will bear this picture in mind in what follows. The intention is to discuss the properties of solutions that resemble (3.14) in the appropriate extension of the theory.

With the above ansatz, the divergence condition (3.9) gives $\partial_{\mu} c=0$ and the metric equation of motion (3.8) reduces to the massless Einstein's equation for $\hat{g}$ with an observable cosmological constant,

$$
\Lambda_{o b s}=\Lambda-6 m^{2}(1-c)
$$

Hence all background solutions are the same as in Einstein-Hilbert gravity with $\Lambda_{\text {obs }}$. However, metric fluctuations around this background are massive, with a mass term,

$$
\frac{1}{4} m_{h}^{2} \sqrt{-\hat{g}}\left(h_{\nu}^{\mu} h_{\mu}^{\nu}-\left(h_{\mu}^{\mu}\right)^{2}\right), \quad \quad m_{h}^{2}=m^{2} c .
$$

The indices are raised with $\hat{g}$. This can be read off from the minimal massive action after taking into account $\Lambda_{o b s}$, which is itself read off from the metric equation (not from the action).

Since $c>0$, tuning it can neutralize a positive $\Lambda \leq 6 \mathrm{~m}^{2}$, as well as any negative $\Lambda$. Note that $m^{2}$ need not be small to recover the GR solutions and that the fluctuations are light as long as $c$ is small. In the extreme case, $m^{2}$ could be of the same order as a quantum field theory $\Lambda$ (say, at TeV scale) and $c<<1$ to cancel a large cosmological constant with effectively light gravitons. In the special case of $\Lambda=6 \mathrm{~m}^{2}$, one ends up with a graviton mass of the same order as the observed cosmological constant, $\Lambda_{\text {obs }} \sim 6 m_{h}^{2}$. The ability to cancel a $\Lambda$ in this way generalizes the mechanism of screening of cosmological constant, discussed for a flat spacetime in [33, 29, 22, 12], to any background sourced by any $T_{\mu \nu}$. What is more, since the classical solutions are the same as in GR, the light bending discrepancy between massless and massive gravity is automatically avoided for any source, without invoking the Vainshtein mechanism (although this mechanism may still apply to the fluctuations).

In [34, 35] the authors considered massive graviton excitations over an FRW solution of massless general relativity. They studied the ghost related stability bounds on the Hubble parameter in terms of the graviton mass, generalizing earlier work on massive gravity in de Sitter spacetime [36]. It is easy to see that the ansatz (3.14) with $\hat{g}$ an FRW metric, provides precisely the non-linear framework in which the set up of [34, 35] is realized. Of course, for the reasons mentioned above, to get a consistent picture, one has to include appropriate dynamics for $f_{\mu \nu}$. Then the theory should provide a consistent description beyond the regime of classical stability breakdown [35].

A purpose of this discussion is to emphasize that the relevance of vDVZ discontinuity [8, 9] to the observational viability of massive gravity depends on the choice of $f_{\mu \nu}$. In 
general, if this tensor is close enough to $g_{\mu \nu}$ for a certain classical solution of the massive theory, this $g_{\mu \nu}$ in turn can be very close to the corresponding solution in the massless theory. Thus the observational constraints imposed by the vDVZ discontinuity can be circumvented without invoking the Vainshtein mechanism ${ }^{11}$. This also underlines the need for further investigating the dynamics of $f_{\mu \nu}$ which is beyond the scope of this paper.

\section{The General non-Linear Massive Action}

In this section we show that the minimal massive action of the previous section can be generalized to, at most, a two-parameter family of non-linear actions. The generalizations are constructed in terms of a deformed determinant and correspond to adding interactions to the minimal theory. We elaborate on the formal structure of these terms. The generalized actions are ghost free at least to lowest orders in an expansion around flat space and for a flat auxiliary metric $f_{\mu \nu}$. Hence any ghost free theory of massive gravity must belong to this class.

\subsection{The generalized mass term as a deformed determinant}

The massive action (3.4) is the minimal deformation of $\operatorname{det} \sqrt{g^{-1} f}$ that satisfies the two criteria listed in the beginning of section 3: (i) it is free of ghosts at the lowest order in the fields when perturbing around flat space and (ii) it reduces to the covariant Fierz-Pauli mass term at lowest order in $H_{\nu}^{\mu}$. Let us now consider the most general deformation of the determinant with deformation parameters $\alpha_{n}$. Taking $\sqrt{g^{-1} f}=\mathbb{K}+\mathbb{1}$ we define, ${ }^{12}$

$$
\widehat{\operatorname{det}} \sqrt{g^{-1} f}=\widehat{\operatorname{det}}(\mathbb{1}+\mathbb{K})=\sum_{n=0}^{4} \frac{-\alpha_{n}}{n !(4-n) !} \epsilon_{\mu_{1} \cdots \mu_{n} \lambda_{n+1} \cdots \lambda_{4}} \epsilon^{\nu_{1} \cdots \nu_{n} \lambda_{n+1} \cdots \lambda_{4}} \mathbb{K}_{\nu_{1}}^{\mu_{1}} \cdots \mathbb{K}_{\nu_{n}}^{\mu_{n}} .
$$

The ordinary determinant corresponds to $\alpha_{n}=1$. For future convenience we note that the deformed determinant (4.1) can be rearranged to give an equivalent expression in terms of $\sqrt{g^{-1} f}$, rather than $\mathbb{K}$,

$$
\widehat{\operatorname{det}} \sqrt{g^{-1} f}=\sum_{r=0}^{4} \frac{-\beta_{r}}{r !(4-r) !} \epsilon_{\mu_{1} \cdots \mu_{r} \lambda_{r+1} \cdots \lambda_{4}} \epsilon^{\nu_{1} \cdots \nu_{r} \lambda_{r+1} \cdots \lambda_{4}}\left(\sqrt{g^{-1} f}\right)_{\nu_{1}}^{\mu_{1}} \cdots\left(\sqrt{g^{-1} f}\right) \underset{\nu_{r}}{\mu_{r}}
$$

where,

$$
\beta_{r}=(4-r) ! \sum_{n=r}^{4} \frac{(-1)^{n+r}}{(4-n) !(n-r) !} \alpha_{n} .
$$

It will often be easier to manipulate the deformed determinant in this form.

The claim is that, in terms of the deformed determinant, the full non-linear action is given by,

$$
S=-M_{p}^{2} \int d^{4} x \sqrt{-g}\left[R-2 m^{2} \widehat{\operatorname{det}}\left(\sqrt{g^{-1} f}\right)+\Lambda^{\prime}\right] .
$$

\footnotetext{
${ }^{11}$ Although the massive fluctuations in such backgrounds may still exhibit the vDVZ discontinuity, they will not affect light bending. An exception is massive gravity in AdS backgrounds which is known to not exhibit the vDVZ discontinuity at all [37, 38].

${ }^{12}$ For the curved space $\epsilon$-tensors, we use the conventions of Wald in General Relativity [39], page 432.
} 
This action automatically satisfies the two criteria specified above: At the lowest order in the metric perturbation around flat space, and for $\bar{f}_{\mu \nu}=\eta_{\mu \nu}$, we have $\left.\mathbb{K}^{\mu}{ }_{\nu}\right|_{h=0}=\partial^{\mu} \partial_{\nu} \pi$. Then (4.1) reduces to the most general total derivative term for $\pi$, insuring the absence of ghosts at this order. Also, the generalized mass term contains the minimal mass term (3.4) for the right set of parameters and hence reduces to the covariant Fierz-Pauli action (2.5).

The five coefficients $\alpha_{n}$ (or equivalently $\beta_{n}$ ) in fact represent only two free parameters, besides the graviton mass $m$ and the cosmological constant $\Lambda$. The lowest order term $\alpha_{0}$ can be absorbed into a cosmological constant as was done in (3.5). For clarity, we will define $\alpha_{0}$ in such a way that flat spacetime can be considered a valid background when the cosmological constant $\Lambda$ is zero. This means that the mass term given by the deformed determinant should contain no terms linear in the metric fluctuation $h$. This is equivalent to setting $\alpha_{0}=\alpha_{1}$. Then the total effective cosmological constant is given by $\Lambda$.

A second coefficient can be fixed by the appropriate definition of the graviton mass $m^{2}$. That is, we define the $\alpha_{n}$ such that the term quadratic in $h_{\mu \nu}$ becomes the FP mass term (2.1). This is equivalent to setting $\alpha_{2}-\alpha_{0}=1$. A third coefficient can be eliminated by observing that since the undeformed determinant $\operatorname{det} \sqrt{g^{-1} f}$ does not contribute to the equations of motion it can be used to subtract off one term of the deformed expansion. We will show this below. Thus the generalized action depends on only two free parameters due to its anti-symmetric structure. The deformed determinant does not allow for any further generalization. The recursion relation constructed in [0], and the vanishing of the higher order terms arising from it, can be understood in terms of this structure, as will be explained below.

The proof of the absence of ghosts to quartic order in non-linearities reduces to the calculation given in [0] and will not be repeated here. The presence of the square-root matrix complicates the analysis of the general case, but there are indications that the ghost analysis can be extended to higher orders in an ADM formalism. The general action in the form (4.4) also allows one to go beyond the flat auxiliary metric and consider classical solutions for more general $f_{\mu \nu}$. This allows for a richer class of solutions, however, now the absence of ghost has to be checked separately for different choices of $f_{\mu \nu}$. The structure of the general massive action will be discussed in more detail below.

\subsection{Formal construction}

That (4.4) is the most general massive gravity action needs a little more clarification. The deformed determinant contains terms that are at most fourth order in $\sqrt{g^{-1} f}$. It is natural to ask whether the Lagrangian can contain higher order terms. These terms would have to vanish identically when evaluated at $h_{\mu \nu}=0$ in order to be consistent with the ghostfree construction. The epsilon tensor structure of the deformed determinant above would suggest that no such terms exist. However, in [7] a recursion relation is presented that might seem to allow one to construct higher order terms in the Lagrangian. In what follows we will show explicitly that these higher order terms are identically zero in 4 dimensions.

The vanishing of these terms can be simply understood in the following way: Writing

$\operatorname{det}(\mathbb{1}+\mathbb{X})$ as $e^{\operatorname{Tr} \ln (\mathbb{1}+\mathbb{K})}$ for a general matrix $\mathfrak{X}$, and expanding the exponential in powers of 
$\mathbb{X}$ gives an infinite number of terms. For a $4 \times 4$ matrix, only terms to order 4 are non-zero by the definition of determinant. These terms appear in the construction (4.1). The higher order terms would contribute in higher dimensions, but vanish for $d=4$. These are the terms constructed by the recursion relation given below in (4.8).

We start by considering a general $N \times N$ matrix $\mathfrak{X}$. The determinant of $\mathbb{1}+\mathfrak{X}$ can be written in terms of the eigenvalues $\lambda_{i}$ of the matrix $\mathbb{X}$,

$$
\operatorname{det}(\mathbb{1}+\mathbb{X})=\prod_{i=1}^{N}\left(1+\lambda_{i}\right)=\sum_{k=0}^{N} e_{k}\left(\lambda_{1}, \ldots, \lambda_{N}\right) .
$$

The terms $e_{k}\left(\lambda_{1}, \ldots, \lambda_{N}\right)$ are elementary symmetric polynomials of the eigenvalues. That is, they are the sum of all distinct products of $k$ distinct $\lambda_{i}$,

$$
\begin{aligned}
& e_{0}\left(\lambda_{1}, \ldots, \lambda_{N}\right)=1 \\
& e_{1}\left(\lambda_{1}, \ldots, \lambda_{N}\right)=\lambda_{1}+\ldots+\lambda_{N}, \\
& e_{2}\left(\lambda_{1}, \ldots, \lambda_{N}\right)=\sum_{i<j} \lambda_{i} \lambda_{j} \\
& \vdots \\
& e_{N}\left(\lambda_{1}, \ldots, \lambda_{N}\right)=\lambda_{1} \lambda_{2} \ldots \lambda_{N}=\operatorname{det} \mathfrak{X}, \\
& e_{k}\left(\lambda_{1}, \ldots, \lambda_{N}\right)=0 \text { for } k>N .
\end{aligned}
$$

The eigenvalues can also be used to represent the trace of powers of the matrix $\mathbb{X}^{m}$. Here we will use square brackets to denote the trace,

$$
\left[\bigvee^{m}\right]=\sum_{i=1}^{N} \lambda_{i}^{m}
$$

Newton's identities give a relationship between the $e_{k}\left(\lambda_{1}, \ldots, \lambda_{N}\right)$ and the trace terms,

$$
e_{k}\left(\lambda_{1}, \ldots, \lambda_{N}\right)=-\frac{1}{k} \sum_{m=1}^{k}(-1)^{m}\left[\mathbb{X}^{m}\right] e_{k-m}\left(\lambda_{1}, \ldots, \lambda_{N}\right) .
$$

These identities can be used to write the polynomials $e_{k}\left(\lambda_{1}, \ldots, \lambda_{N}\right)$ entirely in terms of the traces $\left[\mathbb{K}^{m}\right]$. For a generic $4 \times 4$ matrix one finds,

$$
\begin{aligned}
& e_{0}(\mathbb{X})=1 \\
& e_{1}(\mathbb{X})=[\mathbb{X}] \text {, } \\
& e_{2}(\mathbb{X})=\frac{1}{2}\left([\mathbb{X}]^{2}-\left[\mathbb{X}^{2}\right]\right) \\
& e_{3}(\mathbb{X})=\frac{1}{6}\left([\mathbb{X}]^{3}-3[\mathbb{X}]\left[\mathbb{X}^{2}\right]+2\left[\mathbb{X}^{3}\right]\right), \\
& e_{4}(\mathbb{X})=\frac{1}{24}\left([\mathbb{X}]^{4}-6[\mathbb{X}]^{2}\left[\mathbb{X}^{2}\right]+3\left[\mathbb{X}^{2}\right]^{2}+8[\mathbb{X}]\left[\mathbb{X}^{3}\right]-6\left[\mathbb{X}^{4}\right]\right), \\
& e_{k}(\mathbb{X})=0 \text { for } k>4 \text {. }
\end{aligned}
$$

The $e_{k}(\mathbb{X})$ are exactly the terms that appear in the deformed determinant (4.1) as can be seen from (4.5). Replacing $\mathbb{X}$ by $\mathbb{K}=\sqrt{g^{-1} f}-\mathbb{1}$ and setting $N=4$ we can write,

$$
\widehat{\operatorname{det}} \sqrt{g^{-1} f}=\sum_{n=0}^{4} \alpha_{n} e_{n}(\mathbb{K}) \text {. }
$$


Given this formulation, it is apparent that the recursion relation given in [7] is equivalent to Newton's identities (4.8) with the identification $\mathcal{L}_{\text {der }}^{(n)} \rightarrow n$ ! $e_{n}(\mathbb{K})$. For any $N \times N$ matrix

this same recursion relation gives 0 for $k>N$. Thus the higher order terms in $\sqrt{g^{-1} f}$ that give the correct ghost free structure in the decoupling limit must be zero for $n>4$.

The existence of only two free parameters can also be seen at the level of the perturbative Lagrangian presented in [6]. The first two parameters of that theory can be related to the $\alpha_{n}$ in the deformed determinant as $c_{3}=\frac{1}{6}\left(\alpha_{3}-\alpha_{0}\right)$ and $d_{5}=-\frac{1}{48}\left(\alpha_{4}-\alpha_{0}\right)$. However, using the above results, the terms multiplying $f_{7}$ can be shown to vanish. To see this, note that $e_{5}(\mathbb{X})=0$ for a generic $4 \times 4$ matrix. Then, from Newton's identities, the following combination of terms must be identically zero,

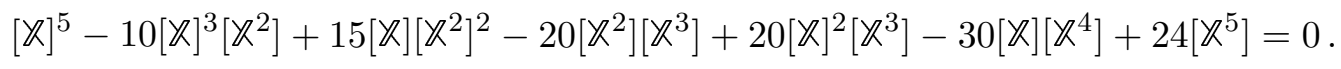

If we take $\mathbb{X}$ to be the matrix $H$, then the coefficient $f_{7}$ in [6] appears in the action multiplying precisely this combination of terms. Analogous arguments can be used to eliminate free parameters that appear at higher orders in $\mathbb{H}$. Thus in four dimensions there are only two free physically meaningful parameters in the massive action. The higher order terms in an $\mathbb{H}$ expansion can be determined entirely from the coefficients $c_{3}$ and $d_{5}$.

\subsection{Equivalent descriptions of the action}

The full non-linear action (4.4) can be written in terms of the $e_{n}(\mathbb{K})$ given by (4.9) above:

$$
S=-M_{p}^{2} \int d^{4} x \sqrt{-g} R(g)+2 M_{p}^{2} m^{2} \int d^{4} x \sqrt{-g} \sum_{n=0}^{4} \alpha_{n} e_{n}(\mathbb{K}) .
$$

With this formalism, it is straight-forward to convert between equivalent descriptions of the action. Let us first make contact with the Lagrangians presented in [20, 6, 7].

To eliminate the redundant parameters we rewrite,

$$
\alpha_{0} e_{0}(\mathbb{K})=\alpha_{0} \operatorname{det} \sqrt{g^{-1} f}-\sum_{n=1}^{4} \alpha_{0} e_{n}(\mathbb{K}) .
$$

The potential term of the action becomes,

$$
2 M_{p}^{2} m^{2} \int d^{4} x \sqrt{-g}\left\{\sum_{n=1}^{4} \bar{\alpha}_{n} e_{n}(\mathbb{K})+\alpha_{0} \operatorname{det} \sqrt{g^{-1} f}\right\},
$$

where $\bar{\alpha}_{n} \equiv \alpha_{n}-\alpha_{0}$. As shown above, the determinant term $\alpha_{0} \operatorname{det}\left(\sqrt{g^{-1} f}\right)$ is nondynamical and can be dropped from the action. In order for flat spacetime to be a valid solution, terms linear in $h$ must vanish in the action when expanded around $\eta_{\mu \nu}$. It follows that $\bar{\alpha}_{1}=0$. Finally, fixing the coefficient of the mass term to be of the canonical form gives $\bar{\alpha}_{2}=1$. The action (4.12) reduces to

$$
S=-M_{p}^{2} \int d^{4} x \sqrt{-g} R(g)+2 M_{p}^{2} m^{2} \int d^{4} x \sqrt{-g}\left(e_{2}(\mathbb{K})+\bar{\alpha}_{3} e_{3}(\mathbb{K})+\bar{\alpha}_{4} e_{4}(\mathbb{K})\right) .
$$


This action has two free parameters, $\bar{\alpha}_{3}$ and $\bar{\alpha}_{4}$. Written in this way it is easy to see that

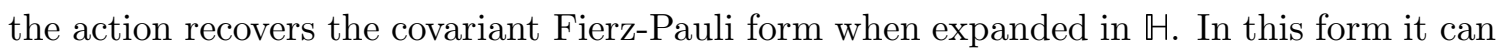
be compared to the results of [20, 6, 7]. When the parameters $\bar{\alpha}_{3}$ and $\bar{\alpha}_{4}$ are set to zero, one obtains the minimal resummed theory presented in [7]. When $\bar{\alpha}_{3}=\bar{\alpha}_{4}=1$ one obtains the minimal deformed determinant action presented above. More generally, the parameters $\bar{\alpha}_{3}$ and $\bar{\alpha}_{4}$ are related to the coefficients $c_{3}$ and $d_{5}$ of [6] by $\bar{\alpha}_{3}=6 c_{3}$ and $\bar{\alpha}_{4}=-48 d_{5}$.

Because of the non-dynamical nature of the det $\sqrt{g^{-1} f}$ term, the action (4.15) has an equivalent description in terms of the lower order $e_{n}$. Moreover, we can use the definitions (4.2) and (4.3) to rewrite the action in terms of $e_{n}\left(\sqrt{g^{-1} f}\right)$ rather than $e_{n}(\mathbb{K})$. When manipulating the action non-linearly it will be more convenient to work with this simpler description. After some rearranging, the action is given by

$$
S=-M_{p}^{2} \int d^{4} x \sqrt{-g} R(g)+2 M_{p}^{2} m^{2} \int d^{4} x \sqrt{-g} \sum_{n=0}^{3} \beta_{n} e_{n}\left(\sqrt{g^{-1} f}\right),
$$

where the $\beta_{n}$ are given by (4.3):

$$
\begin{aligned}
& \beta_{0}=6-4 \bar{\alpha}_{3}+\bar{\alpha}_{4}, \\
& \beta_{1}=-3+3 \bar{\alpha}_{3}-\bar{\alpha}_{4}, \\
& \beta_{2}=1-2 \bar{\alpha}_{3}+\bar{\alpha}_{4}, \\
& \beta_{3}=\bar{\alpha}_{3}-\bar{\alpha}_{4} .
\end{aligned}
$$

This action contains terms that are at most third order in $\sqrt{g^{-1} f}$ rather than fourth order as in 4.15). When expanded in $h_{\mu \nu}$ this action reduces to the basic form of the Fierz-Pauli mass (2.1) at lowest order. The minimal theory presented in the previous section corresponds to the choice of coefficients $\beta_{0}=3, \beta_{1}=-1$ and $\beta_{2}=\beta_{3}=0$ (i.e., $\bar{\alpha}_{3}=\bar{\alpha}_{4}=1$ ). We stress that even though this action does not reduce to the covariant Fierz-Pauli form, the difference is cosmetic and the actions (4.15) and (4.16) are physically equivalent (as long as $\bar{f}_{\mu \nu}$ is non-dynamical).

For completeness we also present here the full non-linear equations of motion. To vary the action we use the relationship,

$$
\delta \operatorname{Tr}\left[\left(\sqrt{g^{-1} f}\right)^{n}\right]=\frac{n}{2} \operatorname{Tr}\left[g\left(\sqrt{g^{-1} f}\right)^{n} \delta g^{-1}\right] .
$$

Then it follows that,

$$
\frac{2}{\sqrt{-g}} \delta\left(\sqrt{-g} e_{n}\left(\sqrt{g^{-1} f}\right)\right)=\sum_{m=0}^{n}(-1)^{m+1} \operatorname{Tr}\left[g\left(\sqrt{g^{-1} f}\right)^{m} \delta g^{-1}\right] e_{n-m}\left(\sqrt{g^{-1} f}\right)
$$

Then the variation of the action gives,

$$
R_{\mu \nu}-\frac{1}{2} g_{\mu \nu} R+\frac{m^{2}}{2} \sum_{n=0}^{3}(-1)^{n} \beta_{n}\left[g_{\mu \lambda} Y_{(n) \nu}^{\lambda}+g_{\nu \lambda} Y_{(n) \mu}^{\lambda}\right]=G_{N} T_{\mu \nu}
$$


where $Y_{(n)} \equiv Y_{(n)}\left(\sqrt{g^{-1} f}\right)$ and for $\boldsymbol{X}=\sqrt{g^{-1} f}$ we have defined (with square brackets again denoting the trace),

$$
\begin{aligned}
& Y_{(0)}(\mathbb{X}) \equiv \mathbb{1}, \\
& Y_{(1)}(\mathbb{X}) \equiv \mathbb{X}-\mathbb{1}[\mathbb{X}] \\
& Y_{(2)}(\mathbb{X}) \equiv \mathbb{X}^{2}-\mathbb{X}[\mathbb{X}]+\frac{1}{2} \mathbb{1}\left([\mathbb{X}]^{2}-\left[\mathbb{X}^{2}\right]\right), \\
& Y_{(3)}(\mathbb{X}) \equiv \mathbb{X}^{3}-\mathbb{X}^{2}[\mathbb{X}]+\frac{1}{2} \mathbb{X}\left([\mathbb{X}]^{2}-\left[\mathbb{X}^{2}\right]\right)-\frac{1}{6} \mathbb{1}\left([\mathbb{X}]^{3}-3[\mathbb{X}]\left[\mathbb{X}^{2}\right]+2\left[\mathbb{X}^{3}\right]\right) .
\end{aligned}
$$

\section{Discussion of results and conclusions}

Our main results are summarized in section 1. Here we discuss some of their implications, and related issues.

How general is the setup? One might wonder about the generality of the non-linear

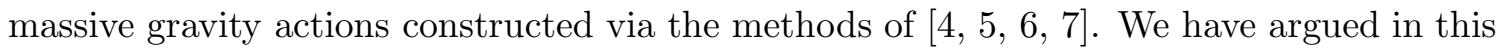
work that in fact these methods are more powerful than they may initially appear, in more than one way. The actions in [4, 5, 6, , 7] are constructed so that they reduce to the covariant FP form (2.5) at the lowest order (quadratic in this case) in $\mathrm{H}$. At first sight, they seem to exclude mass terms that reduce to (3.6), even though these are completely consistent with the basic FP mass (2.1). However, we have shown that the excluded actions differ from the rest only by non-dynamical terms and, in this sense, are not left out. Also, within our formulation the origin of the 2-parameter family of actions, and the impossibility of adding more parameters, becomes manifest.

Moreover, mass terms considered in [4, 5, ,7] and here, depend only on scalar functions of the form $F\left(g^{-1} f\right)$. One may wonder about mass terms that involve scalar functions $F^{\prime}\left(f^{-1} g\right)$. As pointed out in section 2, the quintic order ghost analysis of [6] is general enough to allow for both forms. However, it so happens that at the non-linear level, $F\left(g^{-1} f\right)$ is adequate to express all admissible (by the no-ghost condition) non-linear mass terms in a simple way.

Of course, the theory presented here can be extended by introducing dynamics for $f$, considering additional auxiliary metrics or other such modifications. However, any theory of massive gravity that reduces to the basic FP action at lowest order in $h$, is ghost-free to lowest order in interaction, and depends only on the metric $g$ and the auxiliary metric $f$, must belong to the two-parameter family of actions presented here.

Non-flat auxiliary metric. The ghost analysis of massive gravity actions is valid only for a flat auxiliary metric. The associated constraints introduce deviations from general relativity. For such theory to comply with observations, at the least one needs the Vainshtein mechanism [24, 25]. On the other hand, if one allows the auxiliary metric $f_{\mu \nu}$ to be non-flat, or better, related to $g_{\mu \nu}$ as in section 3.3, then the classical solutions could be very close to general relativity while still retaining some attractive features of massive gravity, like the screening of the cosmological constant. The proper arena of realizing such solutions is theories with dynamical $f_{\mu \nu}$, though not necessarily restricted to bi-gravity 
theories. The ghost issue in such models has to be re-examined. In fact, the recent analysis of massive gravity in FRW spacetimes [34, 35] is relevant to this construction.

The ghost issue. It is clear that the appearance of the square-root matrix $\sqrt{g^{-1} f}$ is an inevitable feature of the Goldstone boson formulation of massive gravity [4]. Such a matrix cannot be easily manipulated analytically. Thus the very setup that simplifies the ghost analysis in the decoupling limit, introduces a complication at the non-linear level. A straightforward ADM analysis of the ghost is therefore not an option, although such an analysis can be carried out for some special cases, for example, for $N_{i}=0$. For more on this, see [7]. (also see the note added below)

Another issue is that even for models with flat $f_{\mu \nu}$, where the ghost analysis applies, the 2-parameter family of actions may need to be further constrained to a single parameter if one is to avoid unstable non-asymptotically flat solutions, as can be seen from [29]. Thus, while the candidate ghost-free actions considered in [6, 7] and here are a step forward, the final question of non-linear massive gravity is far from settled.

Note added: After this work was submitted to the arXiv, [40] appeared with some related results. More recently the absence of ghost at the non-linear level was shown in 44].

\section{Acknowledgments}

We would like to thank Marcus Berg, Cedric Deffayet, Claudia de Rham, Denis Dietrich, Jonas Enander, Gregory Gabadadze, Stefan Hofmann, Eugene Lim, Edvard Mörtsell, Stefan Sjörs, Angnis Schmidt-May, Bo Sundborg, Andrew Tolley and Mikael von Strauss for useful discussions. RAR is supported by the Swedish Research Council (VR) through the Oskar Klein Centre.

\section{References}

[1] M. Fierz, Helv. Phys. Acta 12 (1939) 3.

[2] M. Fierz and W. Pauli, Proc. Roy. Soc. Lond. A 173 (1939) 211.

[3] D. G. Boulware and S. Deser, Phys. Rev. D 6, 3368 (1972).

[4] N. Arkani-Hamed, H. Georgi and M. D. Schwartz, Annals Phys. 305 (2003) 96 [arXiv:hep-th/0210184].

[5] P. Creminelli, A. Nicolis, M. Papucci and E. Trincherini, JHEP 0509, 003 (2005) [arXiv:hep-th/0505147].

[6] C. de Rham and G. Gabadadze, Phys. Rev. D 82, 044020 (2010) [arXiv:1007.0443 [hep-th]].

[7] C. de Rham, G. Gabadadze and A. J. Tolley, arXiv:1011.1232 [hep-th].

[8] H. van Dam and M. J. G. Veltman, Nucl. Phys. B 22 (1970) 397.

[9] V. I. Zakharov, JETP Lett. 12 (1970) 312 [Pisma Zh. Eksp. Teor. Fiz. 12 (1970) 447].

[10] L. Alberte, A. H. Chamseddine and V. Mukhanov, JHEP 1104, 004 (2011) [arXiv:1011.0183 [hep-th]].

[11] G. Gabadadze, Phys. Lett. B 681, 89 (2009) [arXiv:0908.1112 [hep-th]]. 
[12] C. de Rham, Phys. Lett. B 688, 137 (2010) [arXiv:0910.5474 [hep-th]].

[13] M. B. Green and C. B. Thorn, Nucl. Phys. B 367 (1991) 462.

[14] W. Siegel, Phys. Rev. D 49 (1994) 4144 [arXiv:hep-th/9312117].

[15] G. 't Hooft, arXiv:0708.3184 [hep-th].

[16] Z. Kakushadze, Phys. Rev. D 77, 024001 (2008) [arXiv:0710.1061 [hep-th]].

[17] A. H. Chamseddine and V. Mukhanov, JHEP 1008 (2010) 011 [arXiv:1002.3877 [hep-th]].

[18] L. Alberte, A. H. Chamseddine and V. Mukhanov, JHEP 1012, 023 (2010) [arXiv:1008.5132 [hep-th]].

[19] S. F. Hassan and R. A. Rosen, arXiv:1104.1373 [hep-th].

[20] C. de Rham and G. Gabadadze, Phys. Lett. B 693, 334 (2010) [arXiv:1006.4367 [hep-th]].

[21] L. Alvarez-Gaume, D. Z. Freedman and S. Mukhi, Annals Phys. 134, 85 (1981).

[22] S. F. Hassan, S. Hofmann and M. von Strauss, JCAP 1101 (2011) 020 [arXiv:1007.1263 [hep-th]].

[23] L. Berezhiani and M. Mirbabayi, Phys. Rev. D 83, 067701 (2011) [arXiv:1010.3288 [hep-th]].

[24] A. I. Vainshtein, Phys. Lett. B 39, 393 (1972).

[25] C. Deffayet, G. R. Dvali, G. Gabadadze and A. I. Vainshtein, Phys. Rev. D 65, 044026 (2002) [arXiv:hep-th/0106001].

[26] E. Babichev, C. Deffayet and R. Ziour, Phys. Rev. Lett. 103, 201102 (2009) [arXiv:0907.4103 [gr-qc]].

[27] S. Sjörs etal, in preparation.

[28] G. Gabadadze, Talk at the "Return of de Sitter" workshop, March 7-16, 2011, Stockholm, Sweden.

[29] C. de Rham, G. Gabadadze, L. Heisenberg and D. Pirtskhalava, arXiv:1010.1780 [hep-th].

[30] K. Koyama, G. Niz and G. Tasinato, arXiv:1103.4708 [hep-th].

[31] D. Blas, C. Deffayet and J. Garriga, Phys. Rev. D 76 (2007) 104036 [arXiv:0705.1982 [hep-th]].

[32] H. R. Afshar, M. Alishahiha and A. Naseh, Phys. Rev. D 81, 044029 (2010) [arXiv:0910.4350 [hep-th]].

[33] G. Dvali, S. Hofmann and J. Khoury, Phys. Rev. D 76, 084006 (2007) [arXiv:hep-th/0703027].

[34] L. Grisa and L. Sorbo, Phys. Lett. B 686 (2010) 273 [arXiv:0905.3391 [hep-th]].

[35] F. Berkhahn, D. D. Dietrich and S. Hofmann, JCAP 1011 (2010) 018 [arXiv:1008.0644 [hep-th]].

[36] A. Higuchi, Nucl. Phys. B 282, 397 (1987).

[37] M. Porrati, Phys. Lett. B 498, 92 (2001) [arXiv:hep-th/0011152].

[38] I. I. Kogan, S. Mouslopoulos and A. Papazoglou, Phys. Lett. B 503, 173 (2001) [arXiv:hep-th/0011138]. 
[39] R. M. Wald, "General Relativity," Chicago, Usa: Univ. Pr. ( 1984) 491p

[40] T. M. Nieuwenhuizen, arXiv:1103.5912 [gr-qc].

[41] S. F. Hassan and R. A. Rosen, arXiv:1106.3344 [hep-th]. 